\title{
M theory as a holographic field theory
}

\author{
Petr Hořava* \\ California Institute of Technology, Pasadena, California 91125
}

(Received 15 December 1997; published 26 January 1999)

\begin{abstract}
We suggest that $M$ theory could be nonperturbatively equivalent to a local quantum field theory. More precisely, we present a "renormalizable" gauge theory in eleven dimensions, and show that it exhibits various properties expected of quantum M theory, most notably the holographic principle of 't Hooft and Susskind. The theory also satisfies Mach's principle: A macroscopically large space-time (and the inertia of low-energy excitations) is generated by a large number of "partons" in the microscopic theory. We argue that at low energies in large eleven dimensions, the theory should be effectively described by eleven-dimensional supergravity. This effective description breaks down at much lower energies than naively expected, precisely when the system saturates the Bekenstein bound on energy density. We show that the number of partons scales like the area of the surface surrounding the system, and discuss how this holographic reduction of degrees of freedom affects the cosmological constant problem. We propose the holographic field theory as a candidate for a covariant, nonperturbative formulation of quantum $\mathrm{M}$ theory.
\end{abstract}

[S0556-2821(99)01504-0]

PACS number(s): 11.25.-w, 04.60.-m, 04.70.Dy

\section{INTRODUCTION}

$\mathrm{M}$ theory has emerged from our understanding of nonperturbative string dynamics, as a hypothetical quantum theory which has eleven-dimensional supergravity [1] as its lowenergy limit, and is related to string theory via various dualities [2-4] (for an introduction and references, see, e.g., Ref. [5]). While the low-energy effective description of this theory in terms of eleven-dimensional supergravity (coupled to $E_{8}$ Yang-Mills supermultiplets if the space-time manifold has boundaries $[4,6]$ ) is relatively well understood, we still need to clarify how $M$ theory is to be formulated as a nonperturbative quantum theory.

Our search for a nonperturbative formulation of quantum $M$ theory can be guided by some general observations. First of all, $\mathrm{M}$ theory should represent, among other things, a consistent quantum theory of gravity. Using the Bekenstein bound on the maximum entropy in a given region of space [7], 't Hooft and Susskind have argued very convincingly that any such theory should satisfy the holographic principle [8,9] (see also Ref. [10]). The holographic property predicts a radical reduction of the number of degrees of freedom in quantum theory of gravity; unlike in any conventional local field theory, their number should scale like the area surrounding the system.

Other observations come from our improved understanding of nonperturbative string theory. At substringy distances, a new regime of weakly coupled string theory has been discovered and analyzed [11]. In this regime, the short distance physics is dominated by $D 0$-branes, and long-distance gravity is replaced by Yang-Mills gauge theory on world volumes of branes. The matrix theory formulation of quantum M theory, proposed by Banks, Fischler, Shenker, and Susskind $[12,13]$, takes this lesson very seriously and elevates some of the crucial features of $D$-branes to eleven dimensions, using a light-front formulation of $\mathrm{M}$ theory. Sen and

*Email address: horava@ theory.caltech.edu
Seiberg have recently presented an elegant heuristic scaling argument [14], which provides a rationale for the matrix theory proposal and clarifies it significantly.

Matrix theory has proven to be a very impressive candidate for the nonperturbative formulation of $\mathrm{M}$ theory. (For recent reviews, see Ref. [15].) Despite its outstanding successes, however, it still leaves many important questions unanswered. It is background dependent and noncovariant, and the scaling arguments of Ref. [14] suggest the existence of conceptual problems for compactifications on tori of dimension higher than 5 .

In general, it has been suggested that since $M$ theory cannot be a string theory, it must be a new kind of theory, which should perhaps be formulated in terms of completely new degrees of freedom, and require new physical principles. This may even lead to a change in our way of thinking about microscopic physics, perhaps as radical as the discovery of quantum mechanics. (Indeed, a certain amusing analogy between the development of quantum mechanics and that of string theory has been pointed out, see Ref. [16], p. 1.)

We would like to point out a different analogy, which relates the current situation in $\mathrm{M}$ theory to the situation in the theory of strong interactions before the discovery of QCD. In the mid 1960s, it was generally believed that in order to understand strong interactions, local quantum field theory would have to be abandoned altogether, and radically new physical principles would be needed. The efforts to go beyond field theory indeed initiated the development of important new concepts, such as the analytic $S$-matrix, bootstrap, duality, Regge trajectories, etc. However, we know that at the end, the puzzle of the theory of strong interactions turned out to have a beautiful resolution in the "conservative", framework of local quantum field theory.

In this paper we will adopt a similarly "conservative", approach to $M$ theory. Instead of looking for radically new principles and degrees of freedom, we will present evidence suggesting that $M$ theory may in fact be equivalent to a local quantum field theory.

Our starting point in Sec. II will be a Yang-Mills gauge 
theory in eleven dimensions. The gauge group is a certain supersymmetric extension of the eleven-dimensional anti-de Sitter group, but the theory should not be confused with de Sitter supergravity. Microscopically, our theory is a gauge theory, with Yang-Mills gauge symmetries. In addition to the gauge symmetries, we require invariance under space-time diffeomorphisms, as well as parity invariance.

All terms in the Lagrangian that are allowed by the symmetries are of higher order in fields, and are in fact given by Chern-Simons terms. Thus, our theory belongs to the class of Chern-Simons gauge theories [17]. Chern-Simons gravity was first studied in $2+1$ dimensions [18,19], and then extended to higher odd dimensions [20-22]. Our formulation will closely follow that of Refs. $[19,20]$.

When expanded around maximally symmetric solutions, the theory has no propagator, and the low-energy field theory is ill defined, or at least difficult to understand with conventional methods. In Sec. III, we adopt the following effectivetheory approach to this issue. We will not attempt to quantize the theory microscopically. Instead, we will try to identify a low-energy regime, in which the theory does have a conventional low-energy effective field theory description, with excitations propagating in a macroscopically large space-time.

In order to find such a macroscopic low-energy regime, we will have to introduce matter, in the form of firstquantized particles (or "partons") represented by Wilson lines-the only objects that couple naturally to the gauge field. A large space-time will require a large number of partons. We will see that the theory satisfies Mach's principle [23]: Macroscopically large space-times and the inertia of propagating low-energy degrees of freedom will be generated by the distribution of a large number of partons in the theory.

In Sec. IV we study the theory at low-energies in large eleven dimensions. We will show that for the appropriate choice of the gauge group, the flat eleven-dimensional spacetime is a solution of the theory, in a mean field approximation which replaces the effect of individual partons at large distances by a uniform density of partons. We identify the regime which has low-energy degrees of freedom propagating in a large space-time, and argue that the low-energy physics is effectively described by eleven-dimensional supergravity. We demonstrate that this effective supergravity description naturally breaks down at energies much lower than the naively expected Planck scale.

In Sec. V we show that the breakdown of the low-energy effective theory is in accord with the Bekenstein bound on energy density, and that the theory in fact satisfies the holographic principle. More precisely, we demonstrate that the limit of validity of the low-energy effective supergravity description is reached precisely when the energy in any region of characteristic size $L$ equals the mass of the Schwarzschild black hole with radius $L$. We will see that as expected in a holographic theory, the number of partons in the system scales as the area of the surface surrounding the system. At large distances and low energies, the theory is described by low-energy supergravity, and space-time diffeomorphisms are a part of the dynamical gauge group. The realization of the holographic principle in local field theory also sheds some new light on the cosmological constant problem, and we will find indications suggesting that $\Lambda$ may be naturally small in holographic field theory.

The purpose of this paper is to stress some of the unexpected features of the theory, in particular the holographic property, and to set the ground for a more detailed investigation. Our presentation will be rather sketchy, and we will leave out many details and open questions for further study.

\section{THE THEORY}

Consider a gauge field theory in eleven dimensions, defined as follows. Start with an eleven-dimensional manifold $\mathcal{M}$, with coordinates $x^{M}, M=0, \ldots, 10$. Our theory will be a gauge theory described by a Yang-Mills one-form potential $A$, in the adjoint representation of a certain gauge group $\mathcal{G}$. We impose gauge invariance under the Yang-Mills gauge transformations

$$
\delta A_{M}=D_{M} \varepsilon .
$$

There is no preferred metric on $\mathcal{M}$, and we require that the theory be invariant under local diffeomorphisms of $\mathcal{M}$. The only Lagrangian that respects these symmetries is the ChernSimons Lagrangian

$$
\mathcal{L}=-\frac{1}{g^{2}} \int_{\mathcal{M}} \omega_{11}(A),
$$

where $\omega_{11}(A)$ is a Chern-Simons eleven form, defined by

$$
d \omega_{11}(A)=\operatorname{Tr}(F \wedge \cdots \wedge F) .
$$

Here $F$ is the field strength associated with $A$, Tr refers to a symmetric, invariant six-tensor on $\mathcal{G}$. In fact, the Lagrangian can be a linear combination of all possible such terms if there is more than one invariant six-tensor on $\mathcal{G}$ that satisfy all other symmetry restrictions we may want to impose on the theory; each term would then have its own coupling constant $g$. The theory is renormalizable in the elementary sense that all couplings allowed by the symmetries are marginal.

Equation (2.3) can be solved, leading to an explicit formula for the Chern-Simons form $\omega_{11}(A)$ (see, e.g., Ref. [24]),

$$
\begin{aligned}
\omega_{11}(A)= & 6 \int_{0}^{1} d t \operatorname{Tr}\left[A \wedge\left(t d A+t^{2} A \wedge A\right) \wedge \cdots \wedge\right. \\
& \left.\times\left(t d A+t^{2} A \wedge A\right)\right] .
\end{aligned}
$$

The leading term in $\omega_{11}(A)$ is proportional to $\operatorname{Tr}(A \wedge d A \wedge \cdots \wedge d A)$; all other terms are of higher order in $A$.

The coupling constant $g$ in Eq. (2.2) is dimensionless. It may be quantized, depending on the precise choice of the gauge group and $\mathrm{Tr}$. The quantization condition can be derived as follows. Consider a twelve-dimensional manifold $\mathcal{B}$ whose boundary is $\mathcal{M}$, and extend the gauge connection over $\mathcal{B}$. The Lagrangian (2.2) is then more precisely defined using Eq. (2.3), as an integral of $\operatorname{Tr}(F \wedge \cdots F)$ over $\mathcal{B}$ :

$$
\mathcal{L}=-\frac{1}{g^{2}} \int_{B} \operatorname{Tr}(F \wedge \cdots \wedge F) .
$$


The quantization condition on the coupling arises from the requirement that $\mathcal{L}$ be independent of $\mathcal{B}$ and the way $A$ has been extended over $\mathcal{B}$. Typically, this leads to

$$
\frac{1}{g^{2}} \sim k,
$$

with $k$ an integer. ${ }^{1}$

So far we have imposed local diffeomorphism invariance as the only symmetry in addition to local gauge invariance. Our understanding of low-energy effective M theory indicates that any candidate for non-perturbative formulation of $\mathbf{M}$ theory should also be parity invariant. ${ }^{2}$ The $\mathbf{Z}_{2}$ transformation $\mathcal{P}_{0}$ that changes space-time orientation by reversing one of the space-time dimensions (say $x^{1}$ ) cannot be a symmetry of the Chern-Simons gauge theory, since each ChernSimons form is odd under $\mathcal{P}_{0}$. In order to become a symmetry, $\mathcal{P}_{0}$ has to be accompanied by an involution $\mathcal{I}$ on the gauge group $\mathcal{G}$. Depending on the choice of $\mathcal{G}$ and $\mathcal{I}$, the microscopic theory will be constrained by the requirement of invariance under parity, now defined as

$$
\mathcal{P}=\mathcal{P}_{0} \cdot \mathcal{I},
$$

leading to restrictions on admissible $\mathrm{Tr}$ that can appear in Eq. (2.2).

Gauge group and parity invariance. As our gauge group, we will choose a supersymmetric extension of the anti-de Sitter group in eleven dimensions. We need the de Sitter group as a part of the microscopic gauge group, because only in that case we will eventually find a low-energy regime described by effective supergravity with the conventional Lagrangian linear in curvature, and the flat space as a solution of the low-energy theory.

The anti-de Sitter group is generated by $P_{A}$ and $J_{A B}$, with $A, B=0, \ldots, 10$. There is an invariant six-tensor on the anti-de Sitter group that will play crucial role in our theory,

$$
\left\langle P_{A} J_{A_{1} A_{2}} \cdots J_{A_{9} A_{10}}\right\rangle=\epsilon_{A A_{1} \cdots A_{10}}
$$

(with all other terms zero). This six tensor defines a ChernSimons eleven form of the anti-de Sitter group. ChernSimons gravity with this Lagrangian was first studied in various dimensions by Chamseddine [20]. Our Lagrangian will be a supersymmetric extension of this bosonic Chern-Simons Lagrangian.

To make any contact with $\mathrm{M}$ theory, we need at least 32 supercharges. It was shown by van Holten and Van Proeyen in Ref. [27] that the minimal supersymmetric extension of the eleven-dimensional anti-de Sitter group into a supergroup with a 32-component supercharge $Q_{\alpha}$ requires the introduction of an extra bosonic five-form charge $K_{A_{1} \cdots A_{5}}$, which extends the group to $\operatorname{OSp}(1 \mid 32)$.

\footnotetext{
${ }^{1}$ In the case of the de Sitter gauge group, directly relevant to the present paper, the issue of coupling constant quantization has been discussed in Ref. [25].

${ }^{2}$ We know that $\mathrm{M}$ theory is parity invariant [4,26]. Indeed, in $\mathrm{M}$ theory parity can be gauged, leading to the sector of heterotic vacua of the theory.
}

We want to impose parity invariance as a symmetry of our theory. It turns out that the minimal supersymmetric extension OSp $(1 \mid 32)$ of the anti-de Sitter group is not compatible with parity. Indeed, we know how $\mathcal{I}$ should act on the bosonic anti-de Sitter generators: Both $P_{A}$ and $J_{A B}$ flip signs whenever $A$ or $B=1$. On the fermionic generators, $\mathcal{I}$ acts by

$$
Q_{\alpha} \rightarrow\left(\Gamma_{1} Q\right)_{\alpha} .
$$

It is easy to see that $\mathcal{I}$ cannot be extended to an automorphism of $\operatorname{OSp}(1 \mid 32)$. The obstruction comes from the higherform sector of the algebra. It is natural to extend $\mathcal{I}$ to the five-form charge in such a way that it changes sign whenever $A_{i}=1$ for any $i=1, \ldots, 5$. However, this rule does not respect the group structure of $\operatorname{OSp}(1 \mid 32)$, roughly because of the presence of the antisymmetric $\epsilon$ tensor in some of the commutation relations.

Thus, parity invariance will require a non-minimal extension of the anti-de Sitter group, into a group with 64 supercharges. ${ }^{3}$ The minimal choice of the gauge group compatible with parity invariance will contain extra, higher-form bosonic charges $K_{A_{1} \cdots A_{r}}$ for some set of values of $r$, and an extra 32-component supercharge $Q_{\alpha}^{\prime}$. We can now extend the definition of $\mathcal{I}$ to these new charges, requiring that the bosonic charges change sign under $\mathcal{I}$ whenever either of their indices equals 1 , and $Q^{\prime} \rightarrow-\Gamma_{1} Q^{\prime}$. The minimal set of charges that allow commutation relations that respect this $\mathcal{I}$ will contain a six-form, a nine-form, and a ten-form charge, in addition to $P_{A}, J_{A B}$, and $K_{A_{1} \cdots A_{5}}$. (Heuristically, we need a dual charge for each of the original bosonic charges, in order to write down commutation relations without the antisymmetric $\epsilon$ tensor.) These charges generate a group isomorphic to $\operatorname{OSp}(1 \mid 32) \times \operatorname{OSp}(1 \mid 32)$, which happens to be the nonchiral super-Lorentz group in twelve dimensions with signature $(10,2)$ [27]. The bosonic charges form the Lie algebra of $\operatorname{Sp}(32) \times \operatorname{Sp}(32)$. (For details, see Ref. [27].)

We will parametrize the components of the gauge field $A$ in the adjoint of $\operatorname{OSp}(1 \mid 32) \times \operatorname{OSp}(1 \mid 32)$ as follows:

$$
\begin{aligned}
A_{M}= & V_{M}^{A} P_{A}+\frac{1}{2} \omega_{M}^{A B} J_{A B}+\sum_{r} \frac{1}{r !} B_{M}^{A_{1} \cdots A_{r}} K_{A_{1} \cdots A_{r}}+\psi_{M}^{\alpha} Q_{\alpha} \\
& +\eta_{M}^{\alpha} Q_{\alpha}^{\prime}
\end{aligned}
$$

where we have denoted all bosonic higher-form charges collectively by $K_{A_{1} \cdots A_{r}}$, with $r=5,6,9,10$.

Our theory is formally defined by the path integral

$$
\int \mathcal{D} A e^{i \mathcal{L}}
$$

\footnotetext{
${ }^{3}$ First indications that the symmetry algebra underlying elevendimensional supergravity may contain 64 supercharges appeared in Ref. [28]. The importance of algebraic structure in $M$ theory has been stressed by Townsend [29] and Bars [30]. Indeed, 64 supercharges appeared in this algebraic approach to $\mathrm{M}$ theory [30], as a part of the maximal supersymmetric algebra that could contain all string dualities.
} 
We will mostly discuss classical aspects of the theory in this paper, and will not analyze the precise definition of the measure in Eq. (2.11). Our focus will be on an effective approach, and we will try to identify a regime in this microscopic theory where interesting low-energy physics appears already at tree level.

Since the Lagrangian is of higher order in fields, this theory does not have a standard kinetic term; moreover, it is topological in the sense that no metric has been used to write down the theory. Notice that the theory still has dynamical degrees of freedom, as the equations of motion are

$$
F \wedge F \wedge F \wedge F \wedge F=0 .
$$

There is, however, no standard propagator for these local degrees of freedom in the $F=0$ vacuum, nor is there a conventional perturbation theory in terms of weakly coupled localized multiparticle states.

\section{LARGE UNIVERSES AND MACH'S PRINCIPLE}

We live in a large universe, whose behavior at low energies seems well described by a local quantum field theory of particlelike excitations. We want to identify a regime in our theory, which has such a low-energy effective description. In particular, we would like our theory to have an elevendimensional vacuum described at low energies by elevendimensional supergravity, with flat eleven-dimensional space-time as a solution.

\section{A. Effective theory in a large universe}

First of all, we would like to write down the flat spacetime as a particular gauge field configuration. We want to identify the $P_{A}$ component of the gauge field with the vielbein field, and the $J_{A B}$ component with the spin connection. However, the gauge field $A_{M}=V_{M}^{A} P_{A}+\omega_{M}^{A B} J_{A B}+\cdots$ is of dimension 1, while the natural dimension for the vielbein is zero. We introduce the dimensionless vielbein $e_{M}^{A}$, and write

$$
V_{M}^{A}=M e_{M}^{A} .
$$

We will use $\bar{e}_{M}^{A}$ to denote the flat eleven-dimensional vielbein $\bar{e}_{M}^{A}=\delta_{M}^{A}$. Hence, the gauge field configuration that represents the flat eleven-dimensional space-time is

$$
\bar{A}_{M}=M \bar{e}_{M}^{A} P_{A} .
$$

We were able to write down the flat space-time geometry as a particular gauge field $\bar{A}$, at the cost of introducing a mass scale $M$ into the theory. This mass scale is not a part of the path integral definition of our theory. Rather, it appears as a property of the particular gauge configuration $\bar{A}$.

The mass scale $M$ can be interpreted as the inverse characteristic size of the universe (or, more generally, of the box large enough to contain our system). Indeed, the "dimensionless volume" of a ten-dimensional spacelike hypersurface $\mathcal{M}_{10} \subset \mathcal{M}$

$$
\int_{\mathcal{M}_{10}} V \wedge \cdots \wedge V
$$

is a number of order 1 , which gives for the standard volume

$$
\mathcal{V}=\int_{\mathcal{M}_{10}} e \wedge \cdots \wedge e \sim \frac{1}{M^{10}}
$$

Of course, this argument could be easily refined to include the case with a flat metric on $\mathcal{M}_{10}$ of toroidal topology; the radii of the torus would then be measured in units of $L$ $\equiv M^{-1}$.

There are two puzzles that we have have to resolve in our scenario. First, the flat eleven-dimensional space-time (3.2) is not a solution of the classical equations of motion of our $\operatorname{OSp}(1 \mid 32) \times O S p(1 \mid 32)$ Chern-Simons gauge theory. There is a formal solution of the equation of motion, which describes the anti-de Sitter space. However, there is no conventional low-energy effective theory that would result from expanding the microscopic gauge theory around the anti-de Sitter solution. In particular, the formal expansion would have no quadratic term in the Lagrangian, and no propagator for particlelike degrees of freedom. According to the logic of our approach, we are only interested in low-energy regimes that have a conventional effective field theory description.

Another puzzling feature of the theory is the presence of a dimensionless coupling $g$ in Eq. (2.2). We know that M theory-at least at low energies, where it is well described by eleven-dimensional supergravity - does not contain any such free dimensionless parameters. If our theory is to be a reasonable candidate for the microscopic description of $\mathrm{M}$ theory, we have to explain why $g$ does not appear as a free dimensionless coupling in the theory at low energies.

We will see momentarily how both of these issues are resolved when we introduce partonic matter into the theory. The discrete coupling constant $k$ that appears in Eq. (2.6) will turn out to play the role of the number of elementary constituents ("partons") in our system. Only for a large number of partons, our theory will have a low-energy description in terms of supergravity degrees of freedom propagating in a macroscopically large space-time. This relation between the number of partons and the size of the lowenergy world is a first indication that our theory satisfies Mach's principle.

\section{B. Matter and Mach's principle}

The gauge field is a one-form, and it couples naturally to point particles. Consider the Wilson line

$$
W_{\mathcal{R}}(C)=\operatorname{tr}_{\mathcal{R}} P \exp \int_{C} A,
$$

where $\mathcal{R}$ is a representation of the gauge group and $C$ is a certain contour in $\mathcal{M}$. The Wilson line defines an observable in our gauge theory, and one can study physical processes that involve correlation functions of a certain number of such Wilson lines. This is in fact the most natural way of introducing matter in our theory. The Wilson lines correspond to trajectories of particles of matter; their species are in correspondence with the representations of the gauge group. These particles will play the role of "partons" in our microscopic theory. 
Consider now a universe $\mathcal{M}$ with $N$ Wilson lines (or "partons") inside. The partons couple to the gauge fields through their current $\mathcal{I}$, which is a sum of delta functions localized at their corresponding contours $C_{i}$. For $N$ Wilson lines the current is

$$
\mathcal{J}=\sum_{i=1}^{N} j^{a} T_{a} \delta\left(C_{i}\right)
$$

(here $T_{a}$ collectively denotes all generators of the gauge group) and the Lagrangian in the presence of the Wilson lines is modified to

$$
\mathcal{L}=-\frac{1}{g^{2}} \int_{\mathcal{M}} \omega_{11}(A)+\int_{\mathcal{M}} \operatorname{tr}(A \wedge \mathcal{J}) .
$$

Notice that since the group generators $T_{a}$ in Eq. (3.6) are matrices in the representations $\mathcal{R}_{i}$ of the gauge group, their presence in the Lagrangian needs further interpretation. The $T_{a}$ in Eq. (3.6) should be properly interpreted as quantum objects that emerge from the quantization of extra degrees of freedom localized at the contours $C_{i}$. This is of course a procedure standard in gauge theories in general, and in Chern-Simons theories in particular [17,31], and we will not repeat the details here. (See Refs. [19,32] for more details on this construction in the case of $2+1$ Chern-Simons gravity.)

The equations of motion in the presence of $N$ partons no longer require the wedge product of five $F$ 's to vanish. Rather, the flux of the gauge field is tied to the current:

$$
F \wedge \cdots \wedge F=g^{2} \mathcal{J} .
$$

Thus, the partons serve as sources for the field strength flux, which is nonzero and localized at the $N$ contours $C_{i}$, and zero outside the trajectories of the partons.

In the next section, we will be interested in describing such system at large distances, where the collective effect of a large number of Wilson lines can be summarized in terms of a uniform mean field, representing macroscopic spacetime geometry. Our theory is actually an implementation of Mach's principle [23]: The geometry of space-time is generated as a collective effect by the distribution of matter (represented by the partons) in the microscopic theory. The flat microscopic space-time emerges as a collective effect, in the presence of a nontrivial matter distribution. In the absence of matter, not even an empty, flat macroscopic space-time is possible. At low energies, our theory also satisfies Mach's principle in another of its classic formulations: The inertia of propagating particlelike degrees of freedom is generated as a collective effect determined by the distribution of matter in the microscopic theory.

\section{LOW-ENERGY EFFECTIVE SUPERGRAVITY IN ELEVEN DIMENSIONS}

\section{A. Mean field theory and flat eleven-dimensional space-time}

We are interested in the physics at distances much larger than the characteristic distance between two partons. At those distances, we can effectively approximate the source
$\mathcal{J}$-which is microscopically a sum of $N$ delta functions (3.6) — by a uniform density field $J$,

$$
J=c N M^{10} \epsilon_{A_{1} \cdots A_{11}} P^{A_{1}} \bar{e}^{A_{2}} \wedge \cdots \wedge \bar{e}^{A_{11}} .
$$

We expect the mean field approximation to be valid at distances much larger than the characteristic distance between partons as defined a posteriori by $\bar{e}_{M}^{A}$. This approximation is somewhat reminiscent of the average field approximation frequently used in the theory of condensed matter systems described by Chern-Simons theory; see, e.g., Refs. [33,34].

We will adopt this mean field ansatz for the rest of the paper, and will not attempt to derive it from the microscopic theory. In particular, we will not identify precisely the species of partons that leads to the mean field current, leaving this very important point to future study.

In order to write down the mean field ansatz (4.1) for $\mathcal{J}$ in terms of the flat space vielbein $\bar{e}_{M}^{A}$, we had to use the mass scale $M$ that appeared already in Eq. (3.2). This mass scale has been interpreted as the characteristic inverse size of the universe [see Eq. (3.4)]. This interpretation of $M$ is compatible with the mean field theory requirement that the total flux of the uniform density field $J$ be equal to that of the partonic current $\mathcal{J}$,

$$
\int_{\mathcal{M}_{10}} J^{0}=c N
$$

The multiplicative constant $c$ on the right hand side of Eqs. (4.1) and (4.2) is independent of $N$. This constant measures the contribution of an individual parton into $J^{0}$, and will have to be determined a a posteriori due to our lack of knowledge about the precise microscopic origin of Eq. (4.1).

Our theory is defined by Eq. (2.2), with $\operatorname{Tr}$ being the parity-invariant $\operatorname{OSp}(1 \mid 32) \times \mathrm{OSp}(1 \mid 32)$ invariant supersymmetric extension of Eq. (2.8). Due to the presence of the current on the right-hand side of the mean field equations of motion

$$
F \wedge \cdots \wedge F=g^{2} J
$$

the flat eleven-dimensional space

$$
\bar{A}_{M}=M \bar{e}_{M}^{A} P_{A}
$$

is indeed a solution of the theory.

When integrated over the spacelike hypersurface $\mathcal{M}_{10}$, the time component of the equations of motion requires

$$
\int_{\mathcal{M}_{10}} F \wedge \cdots \wedge F=g^{2} \int_{\mathcal{M}_{10}} J
$$

which leads to

$$
\operatorname{cg}^{2} N=1 \text {. }
$$

We choose the value of $c$ (which is independent of $g$ and $N$ ) such that the quantized gauge coupling $k \sim 1 / g^{2}$ is precisely 
equal to $N .^{4}$ In other words, the quantized gauge coupling constant $k \sim 1 / g^{2}$ is to be identified with the number of partons in the system. This resolves one of the puzzles about the low-energy interpretation of our theory-the dimensionless gauge coupling $g$ is determined by the presence of matter in the system.

\section{B. Low-energy field theory}

Now we wish to identify a regime with a well-defined low-energy effective description. At first, our arguments will be independent of the precise supersymmetric extension of the anti-de Sitter group. Therefore, we will study the bosonic anti-de Sitter sector of the theory first, hoping that this will make our arguments more transparent.

Our theory still contains two parameters-a mass scale $M$ introduced in our solution to the mean-field equations of motion, and the dimensionless Chern-Simons coupling that we have just identified with the number of partons $N$ in the system. The requirement that the theory have a low-energy regime described by conventional effective theory will determine one of these parameters in terms of the other.

First we rewrite the theory in terms of rescaled variables suitable for the anticipated low-energy supergravity regime

$$
A_{M}=M e_{M}^{A} P_{A}+\omega_{M}^{A B} J_{A B}+\cdots,
$$

and consider the effective theory for fluctuations near the flat space-time solution. Thus, we assume

$$
e_{M}^{A}-\bar{e}_{M}^{A} \ll 1 \text {. }
$$

It will be convenient to replace the mean-field current $J$ $=N M{ }^{10} P \wedge \bar{e} \wedge \cdots \bar{e}$ by $N M^{10} P \wedge e \wedge \cdots \wedge e$. This corresponds to an improved mean field approximation, in the following sense. The distribution of partons, summarized in the mean field theory by $J$, determines the large-scale metric in spacetime; when we consider geometries $e$ close to but different from the flat geometry $\bar{e}$, the distribution of partons can be expected to adjust to this change of the space-time geometry, leading to the modified mean field expression for $J$ in which $\bar{e}$ is replaced by $e$. Practically, this substitution allows us to keep general covariance in mean field theory.

The bosonic anti-de Sitter sector of our $\operatorname{OSp}(1 \mid 32)$ $\times \operatorname{OSp}(1 \mid 32)$ Lagrangian can be written in terms of the rescaled variables as [20]

\footnotetext{
${ }^{4}$ In more generality, one might consider cases with $k=m N$, with $m$ not necessarily equal to one (but independent of $k$ and $N$ ). Assuming that the theory makes sense for any number of partons, $m$ has to be a positive integer. In fact, this positive integer $m$ relates the number of partons $N$ to the size of the universe they generate, and it might be tempting to refer to it as the "Mach number" of the universe. In this paper, we will only consider universes with Mach number equal to 1 . This is indeed the most refined case-universes with Mach number higher than 1 will have effectively less partons per given volume than the minimal case of Mach number 1, and presumably correspond to partons in higher representations of the gauge group.
}

$$
\begin{aligned}
\mathcal{L}= & -\frac{1}{g^{2}} \int_{\mathcal{M}} \sum_{s=0}^{5} \frac{M^{2 s+1}}{2 s+1}\left(\begin{array}{l}
5 \\
s
\end{array}\right) \\
& \times \epsilon_{A_{1} \cdots A_{11}} e^{A_{1}} \wedge \cdots \wedge e^{A_{2 s+1}} \wedge R^{A_{2 s+2} A_{2 s+3}} \wedge \cdots \wedge R^{A_{10} A_{11}} .
\end{aligned}
$$

$\left(R^{A B} \equiv d \omega^{A B}+\omega^{A C} \wedge \omega_{C}{ }^{B}\right.$ denotes the Riemann curvature of $\left.\omega_{M}^{A B}.\right)$

We are looking for a regime with a well defined lowenergy effective description. In this regime, the low-energy theory should have a kinetic term containing the EinsteinHilbert term linear in $R$. Keeping the Einstein-Hilbert term in Eq. (4.9) finite, we can identify the effective Planck mass

$$
M_{P} \sim \frac{M}{g^{2 / 9}} .
$$

In the low-energy theory, we want to keep $M_{P}$ fixed. Since $g$ is related to the number of partons by Eq. (4.6), the scaling that leads to a well-defined low-energy theory requires $M$ to scale with the number of partons, such that $g$ $\rightarrow 0, M \rightarrow 0$, and $M g^{-2 / 9}$ is fixed. Note that since $M$ is the inverse characteristic size of the universe, this scaling is consistent with the assumption that the universe is macroscopically large in Planck units. Note also that in terms of the microscopic Chern-Simons gauge theory, this regime corresponds to the semiclassical limit $g \rightarrow 0$.

We have identified the low-energy Planck length in terms of the Chern-Simons coupling constant $g$ and the mass parameter $M$. Now we can look more closely at the low-energy effective theory. The Lagrangian (4.9) can be written in terms of $M_{P}$ and $g$ as follows:

$$
\begin{aligned}
\mathcal{L}= & -M_{P}^{9} \int_{\mathcal{M}} \operatorname{Tr}\left(e \wedge \cdots \wedge e \wedge R+\frac{c_{2}}{g^{4 / 9} M_{P}^{2}} e \wedge \cdots \wedge e \wedge R \wedge R\right. \\
& \left.+c_{0} g^{4 / 9} M_{P}^{2} e \wedge \cdots \wedge e+\mathcal{O}\left(g^{-8 / 9} M_{P}^{-4}\right)\right) .
\end{aligned}
$$

[Here, as in Eq. (4.9), the trace is defined by the antisymmetric $\epsilon$ tensor; $c_{0}$ and $c_{2}$ are certain constants of order one and independent of $g$ and $M_{P}$.]

In the effective theory, we will keep only the leading term, proportional to $M_{P}^{9}$ and containing the term linear in curvature. This rule extends to the full $\operatorname{OSp}(1 \mid 32)$ $\times \operatorname{OSp}(1 \mid 32)$ supersymmetric theory, thus leading to a lowenergy supergravity with the Planck mass given by Eq. (4.10). We have also indicated the presence of the cosmological constant term in the bosonic Lagrangian (4.11); this term vanishes in the limit of infinitely large space-time, and should be absent in the full supersymmetric theory. Its dependence on $g$ and $M_{P}$ is of some interest, however, and we will return to this issue briefly in Sec. V.

The effective theory that only keeps terms proportional to $M_{P}^{9}$ can only be valid as long as the higher-order curvature terms in Eq. (4.11) are much smaller than the leading curvature term. Thus, the low-energy supergravity is a good effective theory only at sufficiently large length scales and for sufficiently small space-time curvatures. 
The higher order curvature terms in Eq. (4.11) are indeed suppressed by inverse powers of the Planck mass $M_{P}$. However, powers of $g$ also appear, and we obtain the following condition on the space-time curvature in the effective theory:

$$
R^{A B} \ll g^{4 / 9} M_{P}^{2} .
$$

This is a surprisingly strong restriction on the validity of the low-energy effective field theory. We will see momentarily that this should not be a surprise at all, as our microscopic theory turns out to satisfy the holographic principle. In a holographic theory, the low-energy approximation by an effective field theory in large space-time suffers from a drastic overcounting of the number of degrees of freedom, and therefore should break down much before the naively expected Planckian cutoff. The condition (4.12) is the manifestation of precisely such breakdown of the low-energy effective theory.

\section{Low-energy symmetries: space-time diffeomorphisms}

Microscopically, our theory is a gauge theory. We have seen that at low energies, the theory is effectively described by a Lagrangian linear in Riemann curvature. It is known that this standard (super)gravity Lagrangian is not invariant under the gauge symmetries associated with translations; in supergravity, gauge translations are replaced by diffeomorphisms. In our case, the gauge translations are clearly symmetries of our microscopic theory, and one may wonder how they can get replaced by diffeomorphisms in the effective low-energy theory.

To see how this happens, consider the following. At low enough energies, the higher-curvature terms in the Lagrangian are small, and our theory is described to a good approximation by the low-energy term linear in $R$. The microscopic gauge symmetry algebra can be rewritten in terms of rescaled charges with appropriate dimensions for the lowenergy theory,

$$
P_{A}=M^{-1} \widetilde{P}_{A}, Q_{\alpha}=M^{-1 / 2} \widetilde{Q}_{\alpha} .
$$

Schematically, the relevant part of the commutation relation is

$$
\begin{aligned}
\{\widetilde{Q}, \widetilde{Q}\} & =\Gamma^{A} \widetilde{P}_{A}+\frac{M_{P}}{N^{1 / 9}} \Gamma^{A B} J_{A B}+\Gamma^{A_{1} \cdots A_{5}} K_{A_{1} \cdots A_{5}}+\cdots, \\
{\left[\widetilde{P}_{A}, \widetilde{P}_{B}\right] } & =\frac{M_{P}^{2}}{N^{2 / 9}} J_{A B}+\cdots .
\end{aligned}
$$

(The "ellipses" refer to the higher-form charges.) It is easy to see that even though this is the symmetry algebra of the microscopic theory, it is not a symmetry of the low-energy Lagrangian. Indeed, under gauge translations $\widetilde{\varepsilon}^{A}$, we have from the variation of $e_{M}^{B}$ in the effective Lagrangian.

$$
\delta \mathcal{L}_{\text {eff }} \sim-M_{P}^{9} \int \operatorname{Tr}(\widetilde{\varepsilon} e \wedge \cdots \wedge e \wedge T \wedge R) .
$$

(Here $T^{A}=d e^{A}+\omega^{A}{ }_{B} \wedge e^{B}$ is the torsion of $e$.) In the microscopic theory, this noninvariance is canceled by the variation of a term which is of higher order in curvature, and gauge translations are a gauge symmetry. Indeed, in the microscopic theory we have

$$
\delta_{\widetilde{\varepsilon}} R^{A B} \sim \frac{M_{P}^{2}}{N^{2 / 9}} \widetilde{\varepsilon}^{[A} T^{B]},
$$

and the variation of $R$ in the $R \wedge R$ term cancels that of Eq. (4.15). In the low-energy effective theory, however, the terms of higher order in $R$ are absent, and the gauge translations are not a symmetry. Rather, the effective symmetry algebra of the low-energy theory is related to the contraction of the microscopic algebra, obtained by setting $M_{P} / N^{1 / 9}$ to zero in the commutation relations. In particular, the gauge translations are effectively replaced in the low-energy theory by local diffeomorphisms.

We have argued that the low-energy supergravity description breaks down as we reach curvatures of order $M_{P}^{2} / N^{2 / 9}$. As we approach the limit set by Eq. (4.12), the theory crosses over to an intermediate regime where the mean field approximation should still hold, since the characteristic distance between partons is much smaller than $N^{1 / 9} M_{P}^{-1}$. In that regime, the higher curvature terms become important, and space-time diffeomorphisms are replaced by the microscopic gauge symmetry. In this intermediate regime, the theory becomes a true gauge theory, still in a mean field approximation. $^{5}$

\section{Low-energy supersymmetry}

Having understood how space-time diffeomorphisms appear as a part of the low-energy symmetry, we now return to the full supersymmetric theory. Our discussion will be brief and sketchy. We will not try to demonstrate in detail whether the full low-energy theory really reproduces minimal elevendimensional supergravity of Ref. [1]. We will find indications suggesting that this should be the case, but a more detailed analysis would certainly be desirable.

The full supersymmetry algebra $\operatorname{OSp}(1 \mid 32) \times \mathrm{OSp}(1 \mid 32)$ can be written in terms of the rescaled charges

$$
K_{A_{1} \cdots A_{r}}=M^{-1} \widetilde{K}_{A_{1} \cdots A_{r}}, \quad Q_{\alpha}^{\prime}=M^{-3 / 2} \widetilde{Q}_{\alpha}^{\prime} .
$$

This rescaling is the only one compatible with that of Eq. (4.13) and with the structure of the theory at low energies. The effective symmetry of the low-energy theory is related to the $M \rightarrow 0$ contraction of this microscopic $\operatorname{OSp}(1 \mid 32) \times \operatorname{OSp}(1 \mid 32)$ algebra, for reasons discussed briefly in the previous subsection.

There are several arguments indicating that the lowenergy theory can be expected to reproduce elevendimensional supergravity.

\footnotetext{
${ }^{5}$ Notice that the improved current $N M{ }^{10} e \wedge \cdots \wedge e \wedge P$ is only conserved if torsion is zero. The improved mean field theory in the intermediate regime where $T$ may no longer be zero would require modifications of the improved mean field current that take torsion into account.
} 
(1) The low-energy symmetry algebra obtained from the contraction of the microscopic gauge symmetry is the algebra with 64 supercharges that was identified by D'Auria and Fré in Ref. [28] as the hidden algebra of eleven-dimensional supergravity. (The extra two-form charge that appears in Ref. [28] is to be identified with our $\epsilon_{A B C_{1} \cdots C_{9}} \widetilde{K}^{C_{1} \cdots C_{9}}$, while $K_{A_{1} \cdots A_{6}}$ and $K_{A_{1} \cdots A_{10}}$ decouple in the low-energy algebra.)

(2) In the previous subsection we have seen that in the low-energy theory, $\widetilde{P}_{A}$ acts by diffeomorphisms. Thus, the full low-energy symmetry group is a supersymmetric extension of the diffeomorphism group on $\mathcal{M}$.

(3) Supersymmetry of eleven-dimensional supergravity of course requires the presence of the abelian three-form $C$ in the low-energy spectrum. In the present context, $C$ appears at low energies as a composite field, or more precisely, as a three-form built out of the gauge field $A$. This observation is not new, and was actually one of the main points of Ref. [28]. More details and references on this approach to supergravity can be found in Refs. [35,36].

$C$ is known to be odd under parity, and the explicit formula presented in Ref. [28] that identifies $C$ as a particular composite field certainly satisfies this requirement. Microscopically, there is an obvious candidate for $C$ in the $\mathrm{OSp}(1 \mid 32) \times \mathrm{OSp}(1 \mid 32)$ gauge theory: The Chern-Simons three-form $\omega_{3}(A)$ that is odd under the internal parity $\mathcal{I}$. The microscopic Lagrangian can contain, in addition to the irreducible term $\omega_{11}(A)$, also Chern-Simons terms that are products of lower-dimensional forms, ${ }^{6}$ such as

$$
\int_{\mathcal{M}} \omega_{3} \wedge d \omega_{3} \wedge d \omega_{3}
$$

In the effective theory, this term can be expected to give rise to the supergravity Chern-Simons term $\int C \wedge G \wedge G$, with $G$ $\sim d C$ the field strength of $C$.

It is natural to conjecture that in the low-energy supergravity regime of our theory, the composite field $C$ is the only field that does not decouple from $e_{M}^{A}, \omega_{M}^{A B}$, and $\psi_{M}^{\alpha}$. Given this assumption, the only effective theory of the surviving low-energy degrees of freedom that respects all symmetries is eleven-dimensional supergravity [37].

\section{HOLOGRAPHY}

If our theory is to be a candidate for the microscopic description of $M$ theory, it should be a consistent quantum theory containing gravity. On very general grounds, as argued by 't Hooft and Susskind [8-10], quantum theory of gravity should be expected to satisfy the holographic prin-

\footnotetext{
${ }^{6} \mathrm{Up}$ to this point, we have ignored all such factorizable ChernSimons terms. Such terms can be parity invariant and therefore can indeed appear in the microscopic Lagrangian. However, for our choice of the gauge group, all such parity-invariant terms vanish identically if we set all $B_{M}^{A_{1} \cdots A_{r}}$ and $\eta_{M}$ to zero, and therefore do not affect the main line of arguments of this paper.
}

ciple. In this section we present evidence that our local field theory is indeed holographic.

We have shown above that the Chern-Simons coupling constant $g$ is identified via Eq. (4.6) with the number of partons in the system, while the mass parameter $M$ should be interpreted as the inverse characteristic size of the universe (or, more generally, the inverse characteristic size of the box that is large enough to enclose the system of our interest).

Our system is made out of $N$ partons. Its characteristic size $L$ is given by $M^{-1}$, which can be expressed in Planck units in terms of the number of partons $N$ as (in the order of magnitude)

$$
L=\frac{1}{M}=\frac{1}{g^{2 / 9} M_{P}}=\frac{N^{1 / 9}}{M_{P}} .
$$

In terms of the number of partons $N$ and the Planck scale $M_{P}$, the characteristic volume $\mathcal{V}$ of our system is given by

$$
\mathcal{V} \sim L^{10}=\frac{N^{10 / 9}}{M_{P}^{10}} .
$$

Similarly, the characteristic area $\mathcal{A}$ of the nine-dimensional surface surrounding our system of $N$ partons can be expressed in terms of $N$ and $M_{P}$ as follows:

$$
\mathcal{A} \sim L^{9}=\frac{N}{M_{P}^{9}} .
$$

For the number of partons $N$ in the system we have

$$
N=\left(\frac{M_{P}}{M}\right)^{9} \sim \mathcal{A} M_{P}^{9} .
$$

Thus, the number of partons $N$ in the system scales like the area $\mathcal{A}$ of the nine-dimensional surface surrounding the system, measured in Planck units. In precisely this sense, our theory satisfies the holographic principle.

Note that in order to derive the holographic scaling (5.4), we have only used the quantization condition on the microscopic Chern-Simons coupling constant $g$ that relates $g$ to the number of partons in the theory, in combination with our requirement that the theory have a conventional low-energy limit described by low-energy field theory with a standard kinetic term.

Having seen first indications that our theory is holographic, we can now return to the condition (4.12) that limits the domain of validity of the low-energy effective theory, and demonstrate that this condition is in precise accord with the holographic property of the theory. In a holographic theory, the maximum amount of information and energy in a box of characteristic size $L$ should be limited by the entropy and mass of the black hole with Schwarzschild radius $L$ [7-10].

Consider a configuration in our theory that saturates the inequality in Eq. (4.12). This configuration carries the maximum amount of energy allowed for a configuration in a box of size $L$ by the condition (4.12) that expresses the bound on the validity of the low-energy effective field theory. In the low-energy effective theory, the energy density is given by 


$$
T \sim M_{P}^{9} e \wedge \cdots \wedge e \wedge R
$$

and the total energy in ten-dimensional volume $\mathcal{M}_{10}$ is

$$
E \sim M_{P}^{9} \int_{\mathcal{M}_{10}} e \wedge \cdots \wedge e \wedge R
$$

For the configuration that saturates the inequality in Eq. (4.12), we get

$$
E_{\max } \sim M_{P}^{9} \frac{M_{P}^{2}}{N^{2 / 9}} \int_{\mathcal{M}_{10}} e \wedge \cdots \wedge e
$$

The volume of the universe (or more generally, of the box $\mathcal{M}_{10}$ that contains our system) is $\mathcal{V}=M^{-10}$, which gives for the maximum energy $E_{\max }$

$$
E_{\max } \sim \frac{M_{P}^{11}}{N^{2 / 9}} \frac{1}{M^{10}}=N^{8 / 9} M_{P} .
$$

$E_{\text {max }}$ has a simple form when expressed in terms of the number of partons $N$ and the characteristic inverse size of the box $M$,

$$
E_{\max } \sim N M
$$

This can be further rewritten using the relation (5.4) between the number of partons $N$, the Planck mass $M_{P}$, and the inverse size of the box $M$

$$
E_{\max }=M\left(\frac{M_{P}}{M}\right)^{9}
$$

This is precisely the energy of the Schwarzschild black hole with radius $R_{S}=M^{-1}$.

Thus, the low-energy effective description of the system in terms of conventional supergravity, as derived in the previous section, breaks down when the energy of the system is equal to the mass of the black hole with the Schwarzschild radius equal to the size $M^{-1}$ of the box surrounding the system-precisely as expected in a holographic theory.

Several remarks seem in order.

(1) In addition to the partons represented by the Wilson lines, the microscopic theory contains extra degrees of freedom, in the pure Chern-Simons sector of the theory. Microscopically, there will be fluctuations satisfying the vacuum equations of motion in the space between the Wilson line sources,

$$
F \wedge \cdots \wedge F=0
$$

Could these extra, Yang-Mills degrees of freedom spoil or modify the holographic property of the theory? The answer is no, in the following sense. The holographic property is a property of the low-energy supergravity regime. In the mean

\footnotetext{
${ }^{7}$ This is to be contrasted with the maximum energy expected by the naive Planckian cutoff; indeed, configurations with curvature $R \approx M_{P}^{2}$ would have energy of $\operatorname{order} M_{P}\left(M_{P} / M\right)^{10}$, i.e., Planckian energy per Planckian unit of volume.
}

field approximation, which is valid in large space-time in the supergravity regime, the extra degrees of freedom (5.11) do not play any role-the only low-energy degrees of freedom observable by a low-energy observer are those of the effective supergravity. The theory is holographic, as an effective low-energy theory. ${ }^{8}$

(2) In order to describe local experiments that can be confined inside a box of size $L$, we can stretch the validity of the effective field theory be choosing $M$ as large as possible to still give enough degrees of freedom to describe the experiment, i.e., $M$ should be of order $L^{-1}$ (and not the inverse size of the whole universe). In this way, the holographic property of the theory can be reconciled with the local validity of the low-energy field theory.

(3) As we approach the regime of energies close to the bound (4.12) (which coincides, as we have seen, with the Bekenstein bound), the theory should cross over from the low-energy regime described by eleven-dimensional supergravity to an intermediate regime described by Yang-Mills gauge theory, still in a mean field approximation. According to Eq. (5.9), as we approach the limit of validity of the lowenergy supergravity description, each parton carries energy of order $M$. In the intermediate regime where the theory becomes a gauge theory in the mean field approximation, the excess energy will have to be carried by excited states of the individual partons, or by excitations of the gauge field.

(4) The expression for $E_{\max }$ can be also rewritten as $E_{\text {max }}=M_{P}\left(M_{P} / M\right)^{8}$. This formula suggests that Planckian energy density is actually carried by cells of Planckian size on an eight-dimensional surface. This is reminiscent of the intuitive picture in Ref. [9], with the system being described by some incompressible fluid on the holographic screen. In this picture, the Planckian energy density would be carried by cells of Planckian size in the boundary of such incompressible fluid.

The cosmological constant and naturalness. Since our field theory is a realization of the holographic principle, it might shed new light on the cosmological constant problem. ${ }^{9}$

Looking back at the effective theory (4.11) and ignoring supersymmetry, we do indeed see that the cosmological constant term would be naturally suppressed by a negative power of the number of partons in the system

$$
\Lambda \sim \frac{M_{P}^{11}}{N^{2 / 9}} .
$$

Of course, in the full supersymmetric theory the value of $\Lambda$ would be zero by supersymmetry (and uniqueness of minimal eleven-dimensional supergravity [37]). We have not re-

\footnotetext{
${ }^{8}$ Notice that the theory is holographic precisely to the same extent that it satisfies Mach's principle; macroscopic space-time geometry is determined by the distribution of partons alone, as long as the role of the field-theory degrees of freedom satisfying $F^{5}=0$ is negligible.

${ }^{9}$ The possibility that the cosmological constant problem could be solved in a holographic theory has been stressed repeatedly to the author by Tom Banks. See also Ref. [38].
} 
lied on supersymmetry in our arguments leading to holography, however, and we expect the arguments to hold in vacua with no supersymmetry, or in general, in compactifications to lower dimensions with $\Lambda \neq 0$.

There are indeed two possible points of view in our theory. On one hand, the low-energy field-theory observer underestimates the importance of terms of higher order in curvature and expects them to be suppressed by negative powers of $M_{P}$, and therefore expects the effective supergravity description to be valid for energies up to the Planck scale. The same observer has a naturalness problem with the value of the cosmological constant (5.12), which based on low-energy field theory alone, should be of order $M_{P}^{11}$.

On the other hand, the "microscopic" observer who knows about the underlying Chern-Simons gauge theory has no problem with the small value of the cosmological constant, which is naturally suppressed by an inverse power of the number of partons. This microscopic observer also predicts that the low-energy supergravity description breaks down much faster than expected by the low-energy observer, because the higher curvature terms (and perhaps more importantly, the underlying gauge invariance) become important well before the Planck scale. In holographic field theory, a small cosmological constant seems natural.

This argument will extend to compactifications of the theory to lower dimensions. Consider for example compactifications to four-dimensions on a seven-manifold of volume $L^{7}$. Using Eq. (5.12) and the relation $m_{P}^{2}=M_{P}^{9} L^{7}$ between the four-dimensional Planck mass $m_{P}$ and the elevendimensional Planck mass $M_{P}$ we obtain, for the fourdimensional energy-density $\lambda \sim m_{P}^{2} M^{2}$-an order of magnitude estimate that nicely agrees with the experimental bounds on $\lambda[39]$.

\section{COMMENTS}

In this paper, we have studied a local field theory in eleven dimensions, which contains low-energy supergravity and exhibits the holographic property of 't Hooft and Susskind. We have presented this holographic field theory as a possible candidate for a covariant, "wave mechanics" formulation of nonperturbative quantum $\mathrm{M}$ theory.

In this approach to $\mathrm{M}$ theory, we do not suggest new "fundamental principles" for the microscopic physics at the Planck scale. Instead, our results seem to support the conjecture that $\mathrm{M}$ theory might be well described by an effective field theory, all the way to (and perhaps even beyond) the Planck scale. Such effective field theory may in principle be well-defined to all energy scales (just as QCD is well defined). The expected "low-energy" phenomena (such as eleven-dimensional supergravity and the holographic principle) would emerge hierarchically at lower energies in this effective framework.

We have focused our attention on the minimal theory compatible with the requirements of supersymmetry and parity invariance, which leads to gauge group $\operatorname{OSp}(1 \mid 32) \times \operatorname{OSp}(1 \mid 32)$ with 64 supercharges. In the framework of effective theory, this minimal theory can in principle be embedded into an even larger theory, with bigger super- symmetry algebra. In this respect, the eleven-dimensional superconformal group $\operatorname{OSp}(1 \mid 64)$ would be a particularly natural choice. Whether such an extension will be useful or necessary is unclear.

Perhaps the most surprising result of this paper is the fact that the holographic principle is compatible with microscopic locality. By microscopic locality we mean the fact that the theory is formulated in terms of fields (and possibly a system of partons) with a Lagrangian which is a local function on the underlying eleven-dimensional manifold. Effectively, this microscopic locality can still lead to apparent macroscopic nonlocality, which can manifest itself in the effective low-energy theory in effects such as the holographic property.

One is naturally curious about possible relations of the holographic field theory to matrix theory. We do not have much to say about this issue, except for noticing that it is tempting to compare the partons of the holographic field theory with the $D 0$-brane degrees of freedom of matrix theory. One can formulate the holographic field theory in light-cone gauge, and try to integrate out the gauge field degrees of freedom at low energies. This would leave us with an effective theory of $N$ partons, which could then be compared to matrix theory.

We have studied the theory on manifolds without boundaries. It might be interesting to point out that the anomaly cancellation mechanism $[4,6]$ that predicts the existence of $E_{8}$ super Yang-Mills "edge states" in M theory on manifolds with boundaries bears a remarkable resemblance to the anomaly cancellation mechanism that predicts the existence of similar edge states in Chern-Simons gauge theory $[31,33,34,40,41]$.

The construction presented in this paper can also be repeated in lower space-time dimensions $D=4 p-1$, thus suggesting a possible hierarchy of $\mathrm{M}$ theories in three and seven dimensions. The $(2+1)$-dimensional case is somewhat trivial, but the $(6+1)$-dimensional case might be of more interest. Indeed, here we have an interesting option that does not exist in eleven dimensions: The gauge group can be extended to contain an extra compact group [say $\mathrm{SU}(n)$ ], and we can try to identify regimes in which supergravity decouples in a flat space-time, possibly leaving only $\mathrm{SU}(n)$ degrees of freedom.

The local quantum field theory presented in this paper is described at low energies by supergravity, and satisfies the holographic principle of 't Hooft and Susskind. Regardless of whether or not it will play any role in our future understanding of $\mathrm{M}$ theory, holographic field theory might be an interesting testing ground for questions that originally motivated the formulation of the holographic principle [8,9], most notably the black hole information paradox [10].

\section{ACKNOWLEDGMENTS}

I wish to thank Tom Banks, Itzhak Bars, Eric Gimon, Per Kraus, Christof Schmidhuber, John Schwarz, Lenny Susskind, and Edward Witten for useful discussions at various stages of this work. This work has been supported by DOE Grant No. DE-FG03-92-ER 40701. 
[1] E. Cremmer, B. Julia, and J. Scherk, Phys. Lett. 76B, 409 (1978).

[2] C. M. Hull and P. K. Townsend, Nucl. Phys. B438, 109 (1995); P. K. Townsend, Phys. Lett. B 380, 184 (1995); E. Witten, Nucl. Phys. B443, 85 (1995).

[3] J. H. Schwarz, Phys. Lett. B 367, 97 (1996).

[4] P. Hořava and E. Witten, Nucl. Phys. B460, 506 (1996).

[5] P. K. Townsend, "Four Lectures on M-Theory,", hep-th/9612121.

[6] P. Hořava and E. Witten, Nucl. Phys. B475, 94 (1996).

[7] J. D. Bekenstein, Phys. Rev. D 49, 1912 (1994).

[8] G. 't Hooft, in Salamfestschrift, edited by A. Ali, J. Ellis, and S. Randjbar-Daemi (World Scientific, Singapore, 1993), gr-qc/ 9310026.

[9] L. Susskind, J. Math. Phys. 36, 6377 (1995).

[10] G. 't Hooft, "Quantum Information and Information Loss in General Relativity,' gr-qc/9509050; Int. J. Mod. Phys. A 11, 4623 (1996).

[11] U. H. Danielsson, G. Ferretti, and B. Sundborg, Int. J. Mod. Phys. A 11, 5463 (1996); D. Kabat and P. Pouliot, Phys. Rev. Lett. 77, 1004 (1996); M. R. Douglas, D. Kabat, P. Pouliot, and S. H. Shenker, Nucl. Phys. B485, 85 (1997).

[12] T. Banks, W. Fischler, S. H. Shenker, and L. Susskind, Phys. Rev. D 55, 5112 (1997).

[13] L. Susskind, "Another Conjecture about M(atrix) Theory,", hep-th/9704080.

[14] A. Sen, Adv. Theor. Math. Phys. 2, 51 (1998); N. Seiberg, Phys. Rev. Lett. 79, 3577 (1997).

[15] T. Banks, Nucl. Phys. B (Proc. Suppl.) 67, 180 (1998); D. Bigatti and L. Susskind, "Review of Matrix Theory," hep-th/9712072.

[16] M. B. Green, J. H. Schwarz, and E. Witten, Superstring Theory (Cambridge University Press, Cambridge, England, 1987), Vols. 1 and 2.

[17] E. Witten, Commun. Math. Phys. 121, 351 (1989).

[18] P. van Nieuwenhuizen, Phys. Rev. D 32, 872 (1985); A. Achúcarro and P. K. Townsend, Phys. Lett. B 180, 89 (1986).

[19] E. Witten, Nucl. Phys. B311, 46 (1988); B323, 113 (1989).

[20] A. H. Chamseddine, Phys. Lett. B 233, 291 (1989); Nucl. Phys. B346, 213 (1990).

[21] M. Bañados, C. Teitelboim, and J. Zanelli, Phys. Rev. Lett. 69, 1849 (1992); Phys. Rev. D 49, 975 (1994); Phys. Rev. Lett. 72, 957 (1994).

[22] M. Bañados, R. Troncoso, and J. Zanelli, Phys. Rev. D 54,
2605 (1996); R. Troncoso and J. Zanelli, ibid. 58, 101703 (1998).

[23] Mach's Principle: From Newton's Bucket to Quantum Gravity, edited by J. B. Barbour and H. Pfister (Birkhaüser, Boston, 1995).

[24] J. A. de Azcárraga and J. M. Izquierdo, Lie Groups, Lie Algebras, Cohomology and Some Applications in Physics (Cambridge University Press, Cambridge, England, 1995).

[25] J. Zanelli, Phys. Rev. D 51, 490 (1995).

[26] E. Witten, J. Geom. Phys. 22, 1 (1997).

[27] J. W. van Holten and A. van Proeyen, J. Phys. A 15, 3763 (1982).

[28] R. D’Auria and P. Fré, Nucl. Phys. B201, 101 (1982).

[29] P. K. Townsend, " $p$-Brane Democracy,"' hep-th/9507048; “'M-Theory from its Superalgebra,', hep-th/9712004.

[30] I. Bars, Phys. Rev. D 55, 2373 (1997); Phys. Lett. B 403, 257 (1997).

[31] S. Elizur, G. Moore, A. Schwimmer, and N. Seiberg, Nucl. Phys. B326, 108 (1989).

[32] S. Carlip, Nucl. Phys. B324, 106 (1989).

[33] A. Zee, 'Quantum Hall Fluids,'” cond-mat/9501022; A. Lopez and E. Fradkin, "Fermionic Chern-Simons Field Theory for the Fractional Quantum Hall Effect,', cond-mat/9704055.

[34] F. Wilczek, Fractional Statistics and Anyon Superconductivity (World Scientific, Singapore, 1990); E. Fradkin, Field Theories of Condensed Matter Systems (Addison-Wesley, New York, 1991).

[35] L. Castellani, P. Fré, F. Giani, K. Pilch, and P. van Nieuwenhuizen, Ann. Phys. (N.Y.) 146, 35 (1983); R. D’Auria, P. Fré, P. K. Townsend, and P. van Nieuwenhuizen, ibid. 155, 423 (1984); P. Fré, Class. Quantum Grav. 1, L81 (1984).

[36] L. Castellani, R. D'Auria and P. Fré, Supergravity and Superstrings: A Geometric Perspective (World Scientific, Singapore, 1991), Vols. 1-3.

[37] K. Bautier, S. Deser, M. Henneaux, and D. Seminara, Phys. Lett. B 406, 49 (1997).

[38] T. Banks, "SUSY Breaking, Cosmology, Vacuum Selection and the Cosmological Constant in String Theory," hep-th/9601151.

[39] S. Weinberg, Rev. Mod. Phys. 61, 1 (1989).

[40] S. Carlip, Phys. Rev. D 55, 878 (1997).

[41] A. P. Balachandran, L. Chandar, and E. Ercolessi, Int. J. Mod. Phys. A 10, 1969 (1995); A. P. Balachandran, L. Chandar, and Arshad Momen, Nucl. Phys. B461, 581 (1996). 